Revista de Psicología Vol. 29 (2), 2011 (ISSN 0254-9247)

\title{
Propiedades psicométricas de la Escala MESSY (versión autoinforme) en nińos argentinos ${ }^{1}$
}

\author{
María Julia Ipiña ${ }^{2}$ y Leonardo Molina ${ }^{3}$ \\ Universidad Nacional de Córdoba, Argentina \\ Cecilia Reyna ${ }^{4}$ \\ Universidad Nacional de Córdoba / CONICET, Argentina
}

Se evalúan las propiedades psicométricas de la versión de Autoinforme de la Escala Matson para la Evaluación de Habilidades Sociales con Jóvenes (Matson, Rotatori \& Helsel, 1983), que permite evaluar habilidades sociales específicas implicadas en comportamientos adaptativos y no adaptativos. Se estudia la estructura factorial y consistencia interna en una muestra de niños cordobeses $(n=578)$ con edades entre los 7 y 13 ańos $(M=9.48, D E$ $=1.47$ ), de ambos sexos (52.77\% varones). Se analizaron los datos y variables de manera exploratoria, y luego se analizó la estructura factorial. La estructura de 5 factores resultó la más simple y teóricamente coherente: Agresividad/Conducta Antisocial, Habilidades Sociales Apropiadas, Amistad, Sobreconfianza/Celos/Soberbia y Soledad/Ansiedad Social. Los índices de consistencia interna fueron buenos o aceptables en la mayoría de dimensiones. Las diferencias de sexo en las distintas dimensiones y en la puntuación total ofrecen evidencia de la validez del instrumento.

Palabras clave: habilidades sociales, psicometría, niños.

Psychometric properties of the MESSY Scale (self-assessment) in Argentinean children This study examines the psychometric properties of the Matson Evaluation of Social Skills with Youngsters (Matson, Rotatori \& Helsel, 1983), which assesses specific social skills involved in adaptive and non-adaptive behaviors. The factor structure and internal consistency were studied in a sample of children from Cordoba $(n=578)$ of 7 to 13 years old $(M$ $=9.48, S D=1.47)$ of both sexes $(52.77 \%$ males $)$. An exploratory study of data and variables was conducted; then the factor structure was studied. Results suggest that the structure of five factors was the simplest and theoretically most consistent: Aggressiveness/Antisocial Behavior, Appropriate Social Skills, Friendship, Overconfidence/Jealousy/Pride and Loneliness/Social Anxiety. The internal consistent coefficients were good or acceptable in most dimensions. Gender differences observed in the dimensions and total score provide evidence of the validity of the instrument.

Keywords: Social skills, psychometric, children. 

El desempeño social en edades tempranas tiene consecuencias no solo inmediatas en el desarrollo general del nińo, sino también a largo plazo. El comportamiento social competente se relaciona de manera positiva con el rendimiento académico y la autoestima, en tanto que los problemas de conducta generalmente se asocian con abandono escolar, altos niveles de depresión y ansiedad, abuso de drogas en la adolescencia, pobres resultados laborales y relaciones amorosas conflictivas durante la adultez (Parker \& Asher, 1987; Rubin et al., 2004; Schaeffer, Petras, Ialongo, Poduska \& Kellam, 2003).

$\mathrm{Si}$ bien existen numerosas definiciones sobre competencia social, la mayoría de los investigadores reconocen que implica la ejecución de un repertorio de conductas socialmente adecuadas que permiten alcanzar la efectividad en las interacciones sociales, en estrecha relación con estructuras motivacionales y afectivas (Cummings, Kaminski \& Merrell, 2008; Rose-Krasnor, 1997; Trianes, Muñoz \& Jiménez, 1997). Mientras, las habilidades sociales son comportamientos sociales específicos que se manifiestan en la vida diaria y que contribuyen en forma decisiva para alcanzar buenos resultados en las relaciones interpersonales (Pereira Del Prette \& Del Prette, 2002). Cabe resaltar el componente evaluativo del término competencia social, el cual implica la valoración del desempeńo social de un individuo por parte de otros o uno mismo (Gresham, Sugai \& Horner, 2001). Tanto la competencia social como las habilidades sociales se enmarcan dentro del comportamiento adaptativo, mientras que los comportamientos no adaptativos

1 Una versión parcial de este trabajo fue publicada en las Memorias del II Congreso Internacional de Investigación y Práctica Profesional en Psicología, XVII Jornadas de Investigación y Sexto Encuentro de Investigadores de Psicología del MERCOSUR (2010).

2 Licenciada en Psicología. Contacto: julipina83@hotmail.com

3 Licenciada en Psicología. Contacto: leonardomolina940@hotmail.com

4 Licenciada y Doctoranda en Psicología. Contacto: Enrique Barros y Enfermera Gordillo, Ciudad Universitaria, (5000) Córdoba, Argentina; ceciliareyna@gmail.com 
comprenden problemas externalizantes como agresión, pelea y actingout, y problemas internalizantes, como ansiedad, aislamiento social y depresión.

Las técnicas de evaluación del comportamiento social más utilizadas son los cuestionarios, comprendiendo medidas de autoinforme y evaluación de padres o maestros. En una reciente revisión, Matson y Wilkins (2009) inspeccionaron 40 tests de habilidades sociales para niños, y observaron que los instrumentos de mayor uso son la Matson Evaluation of Social Skills with Youngsters (MESSY, Escala Matson de Evaluación de Habilidades Sociales con Jóvenes, Matson, Rotatori \& Helsel, 1983) y el Social Skills Rating System (SSRS, Sistema de Evaluación de Habilidades Sociales, Gresham \& Elliott, como se citó en Matson \& Wilkins, 2009). El MESSY se utiliza con niños de edad escolar y adolescentes, siendo frecuente su uso con poblaciones de individuos bipolares, autistas y con deficiencia visual (Goldstein, Miklowitz \& Mullen, 2006; Matson, Stabinsky-Compton \& Sevin, 1991; Sharma, Sigafoos \& Carroll, 2000).

La evaluación de la competencia social puede implicar auto o hetero-evaluación. Sin duda, la evaluación por parte de agentes externos tiene ciertas ventajas como, por ejemplo, la valoración del comportamiento social de niños pre-verbales y un criterio comparativo menos sesgado. En particular, se destaca el valor del informe de los docentes, el cual ha mostrado ser un mejor predictor de problemas delictivos en la adolescencia y adultez (Bank, Duncan, Patterson \& Reid, 1993) con relación al informe de los padres. Como seńalan Ipińa, Molina y Reyna (2010), el recurrir a los docentes como informantes clave se hace bajo el supuesto de que estos cuentan con una considerable experiencia valorativa del desempeño social infantil. Sin embargo, resulta importante utilizar también medidas de autoinforme, ya que las mismas implican una serie de procesos que sustentan la capacidad de informar sobre la propia conducta y de valorarla según la retroalimentación producida en los demás y el logro de los objetivos; como señalan Trianes, Blanca, García y Sánchez (2003), esta evaluación será la fuente del desarrollo de la autoestima, las expectativas de éxito y la construcción del Yo. 
Si bien la escala MESSY se creó en lengua inglesa, ha sido traducida a otras lenguas, adaptada y utilizada en otros países, como Australia (Spence \& Liddle, 1990), China (Chou, 1997), España (Méndez, Hidalgo \& Inglés, 2002; Trianes, Blanca, Muñoz, Cardelle-Elawar \& Infante, 2002) y Brasil (Martins Teodoro, Käppler, Martins de Freitas, Lima Rodrigues \& Geraldi Haase, 2005), entre otros. Sin embargo, existen diferencias sobre la estructura factorial que explica mejor los datos, observándose distintos números de factores o dimensiones, comprendiendo diferentes ítems. Entre los diversos factores explicativos de tales divergencias se destaca el componente cultural implicado en el comportamiento social (y su evaluación). La versión original de la escala MESSY autoinforme de Matson et al. (1983) comprende cinco dimensiones: habilidades sociales apropiadas, asertividad inapropiada, impulsividad, sobreconfianza y celos/soledad. Asimismo, esa estructura coincide con la versión espańola presentada por Trianes et al. (2002). Mientras que Méndez et al. (2002), en una muestra de adolescentes españoles, observaron una estructura de cuatro dimensiones: agresividad/conducta antisocial, habilidades sociales/asertividad, arrogancia/soberbia y soledad/ansiedad social. Por otra parte, la escala muestra aceptables propiedades de consistencia interna, con índices alfa de de Cronbach de .54 a .80 (Matson et al., 1983) y de .63 a .91 (Méndez et al., 2002).

En nuestro medio, desde hace unos años se cuenta con un par de instrumentos para valorar el comportamiento social en el marco adaptativo. Particularmente, la Escala de Comportamiento Asertivo adaptada y normalizada por Brussino (2002) en niños de 6 a 12 años, la Escala de Habilidades Sociales para nińos preescolares construida a partir de ítems de diversos cuestionarios elaborada por Lacunza, Castro Solano y Contini (2009), la Escala de Comportamiento Preescolar y Jardín Infantil de Merrell estudiada por Reyna y Brussino (2009), y la Escala MESSY versión docente analizada por Ipińa et al. (2010). Además, Schulz (2008) examinó las características de confiabilidad y validez de la escala MESSY en una muestra de nińos de Entre Ríos y Buenos Aires, con nińos y padres como informantes; sin embargo, se desconocen los detalles de las dimensiones encontradas de la escala. 
A partir de estos antecedentes y teniendo en cuenta la relevancia de contar con instrumentos locales con adecuadas propiedades psicométricas para valorar el comportamiento social infantil, este estudio se propuso analizar la estructura factorial y confiabilidad (consistencia interna) de la escala MESSY versión autoinforme en una muestra de nińos cordobeses (Argentina). Asimismo, se examinaron las diferencias de sexo en las dimensiones y puntuación total de la escala.

\section{Método}

\section{Participantes}

Participaron 596 nińos que asistían a instituciones públicas y privadas de las ciudades de Villa María y Córdoba, cuyos padres brindaron el consentimiento informado vía escrita. Se excluyeron 18 niños por presentar datos insuficientes (solo completaron un par de ítems). De esta manera, la muestra quedó conformada por 578 nińos con edades comprendidas entre los 7 y 13 ańos $(M=9.48, D E=1.47), 52.77 \%$ varones.

\section{Instrumento}

Se empleó la versión en español de Trianes et al. (2002) de Matson Evaluation of Social Skills with Youngsters (MESSY) (Matson et al., 1983, Evaluación Matson de Habilidades Sociales con Jóvenes). La escala permite evaluar habilidades sociales específicas implicadas en comportamientos sociales adaptativos, como también comportamientos no adaptativos, considerando la relación con los pares y adultos, y se aplica a niños y jóvenes de 4 a 18 ańos. Posee versiones de autoinforme y evaluación externa (padres y docentes); en este estudio solo se trabajó con la versión de autoinforme, la cual posee 62 ítems y se evalúa con una escala tipo Likert de 4 puntos: $1=$ Nunca, $2=A$ veces, $3=A$ menudo, $4=$ Siempre. Mayor puntuación indica un nivel más alto de comportamientos inadecuados (dimensiones de comportamientos negativos) o adecuados socialmente (dimensiones de comportamientos positivos), en 
tanto que en la puntuación total refleja mayor inadecuación social. El puntaje de los ítems de cada dimensión positiva se deduce del máximo valor posible para esa dimensión y se suma a las dimensiones negativas.

Previo a la recolección de datos, se realizó un estudio piloto con el objetivo de valorar la claridad semántica y sintáctica de la versión de autoinforme bajo análisis. Para ello se aplicó la escala a 15 niños de entre 7 y 13 años de edad. Asimismo, se solicitó a cuatro docentes de $3^{\circ}$ a $6^{\circ}$ grado que revisaran el instrumento con respecto al vocabulario empleado. De esa manera, se obtuvieron las valoraciones de los participantes, que se plasmaron en sutiles modificaciones de la versión presentada por Trianes et al. (2002).

\section{Procedimiento}

Primero se contactó a las autoridades institucionales y luego se solicitó el consentimiento por escrito de padres y alumnos; en el mismo se informó los objetivos del estudio y el carácter anónimo y confidencial de la información brindada, enfatizándose el carácter voluntario de la participación. La aplicación de los cuestionarios se realizó en grupos de cuatro niños, en un aula asignada por la institución para tal fin. Tras la recolección de datos, se llevaron a cabo los análisis estadísticos. Se estudió de manera descriptiva el comportamiento de los ítems y se analizó exploratoriamente la estructura factorial de la escala, estimándose además la consistencia interna. Finalmente, se evaluaron las diferencias de sexo en la puntuación total y las distintas dimensiones de la escala. Los análisis fueron realizados con el paquete estadístico SPSS 17 y FACTOR 7.02.

\section{Resultados}

En primer lugar, se analizó el patrón de valores ausentes a través de la aplicación provista en SPSS 17. El análisis mostró que ninguno de los ítems presentaba más del 5\% de datos ausentes, los cuales se distribuían de manera aleatoria. Debido a ello, se reemplazaron los datos faltantes con los valores correspondientes a la media de cada ítem (Hair, 
Anderson, Tatham \& Black, 1999). Luego, se inspeccionó la existencia de casos atípicos univariados a través del cálculo de puntuaciones estándar. Se consideraron atípicos los casos con puntuaciones $z$ mayores a \pm 3.29 (Tabachnick \& Fidell, 2001). También se examinó la presencia de casos atípicos multivariados, a través de la prueba de distancia de Mahalanobis $(p<.001)$, sin observarse resultados positivos. A partir de esos análisis, se descartaron 11 casos que presentaban puntuaciones extremas, por lo que la muestra quedó integrada por 567 niños.

Posterior a ello se analizó la asimetría y curtosis de los ítems con el fin de detectar los reactivos con valores inadecuados que pudieran afectar la distribución normal de las puntuaciones. Del total de 62 ítems que comprende la escala, 52 ítems presentaron valores de asimetría adecuados $( \pm 1.6)$ y 50 ítems valores adecuados de curtosis ( \pm 1.6$)$, mientras que el resto evidenció valores no tolerables. A pesar de ello, y teniendo en cuenta el carácter exploratorio del análisis, se decidió no descartar los reactivos en este paso con el fin de facilitar comparaciones internacionales. Los resultados descriptivos se muestran en la tabla 1.

\section{Tabla 1}

Media, desviación estándar, asimetría y curtosis de la distribución de los items de la escala MESSY-Autoinforme

\begin{tabular}{lcccc}
\hline Ítem & $M$ & $D E$ & Asimetría & Curtosis \\
\hline Ítem 1 & 2.51 & 0.92 & 0.37 & -0.85 \\
Ítem 2 & 1.22 & 0.45 & 1.89 & 2.74 \\
Ítem 3 & 1.98 & 0.93 & 0.86 & 0.00 \\
Ítem 4 & 1.42 & 0.73 & 1.94 & 3.56 \\
Ítem 5 & 1.74 & 0.81 & 1.06 & 0.75 \\
Ítem 6 & 1.81 & 0.87 & 1.07 & 0.64 \\
Ítem 7 & 1.42 & 0.73 & 1.96 & 3.57 \\
Ítem 8 & 1.49 & 0.85 & 1.79 & 2.34 \\
Ítem 9 & 3.15 & 1.11 & -0.83 & -0.89 \\
Ítem 10 & 3.46 & 0.94 & -1.54 & 0.99 \\
Ítem 11 & 1.90 & 1.01 & 0.94 & -0.20 \\
Ítem 12 & 3.29 & 0.99 & -1.05 & -0.32
\end{tabular}




\begin{tabular}{|c|c|c|c|c|}
\hline Ítem & $M$ & $D E$ & Asimetría & Curtosis \\
\hline Ítem 13 & 3.19 & 1.05 & -0.88 & -0.66 \\
\hline Ítem 14 & 1.47 & 0.78 & 1.83 & 2.87 \\
\hline Ítem 15 & 1.67 & 0.95 & 1.36 & 0.77 \\
\hline Ítem 16 & 3.24 & 1.05 & -1.00 & -0.47 \\
\hline Ítem 17 & 1.50 & 0.83 & 1.71 & 2.16 \\
\hline Ítem 18 & 2.00 & 1.03 & 0.83 & -0.43 \\
\hline Ítem 19 & 1.64 & 0.85 & 1.34 & 1.12 \\
\hline Ítem 20 & 2.52 & 1.19 & 0.07 & -1.52 \\
\hline Ítem 21 & 1.71 & 0.94 & 1.28 & 0.69 \\
\hline Ítem 22 & 1.61 & 0.85 & 1.43 & 1.36 \\
\hline Ítem 23 & 2.37 & 1.12 & 0.25 & -1.30 \\
\hline Ítem 24 & 3.46 & 0.96 & -1.56 & 1.01 \\
\hline Ítem 25 & 1.79 & 0.97 & 1.14 & 0.26 \\
\hline Ítem 26 & 1.73 & 0.92 & 1.14 & 0.35 \\
\hline Ítem 27 & 3.22 & 0.98 & -0.74 & -0.99 \\
\hline Ítem 28 & 3.35 & 0.93 & -1.09 & -0.13 \\
\hline Ítem 29 & 1.35 & 0.69 & 2.30 & 5.25 \\
\hline Ítem 30 & 1.46 & 0.74 & 1.79 & 3.09 \\
\hline Ítem 31 & 2.86 & 1.13 & -0.33 & -1.38 \\
\hline Ítem 32 & 2.92 & 1.16 & -0.45 & -1.37 \\
\hline Ítem 33 & 1.78 & 0.93 & 1.05 & 0.21 \\
\hline Ítem 34 & 3.03 & 1.02 & -0.54 & -1.04 \\
\hline Ítem 35 & 1.81 & 0.96 & 1.05 & 0.08 \\
\hline Ítem 36 & 1.54 & 0.84 & 1.58 & 1.73 \\
\hline Ítem 37 & 2.36 & 1.13 & 0.28 & -1.31 \\
\hline Ítem 38 & 1.84 & 0.96 & 1.06 & 0.20 \\
\hline Ítem 39 & 1.34 & 0.77 & 2.38 & 4.88 \\
\hline Ítem 40 & 3.18 & 1.09 & -0.88 & -0.74 \\
\hline Ítem 41 & 2.05 & 0.99 & 0.74 & -0.44 \\
\hline Ítem 42 & 3.18 & 1.02 & -0.79 & -0.80 \\
\hline Ítem 43 & 3.07 & 1.01 & -0.56 & -1.06 \\
\hline Ítem 44 & 3.33 & 1.01 & -1.18 & -0.06 \\
\hline Ítem 45 & 1.81 & 1.02 & 1.07 & -0.07 \\
\hline Ítem 46 & 2.64 & 1.07 & 0.01 & -1.32 \\
\hline
\end{tabular}




\begin{tabular}{lcccc}
\hline Ítem & $M$ & $D E$ & Asimetría & Curtosis \\
\hline Ítem 47 & 2.91 & 1.09 & -0.48 & -1.14 \\
Ítem 48 & 1.80 & 0.97 & 1.12 & 0.25 \\
Ítem 49 & 1.83 & 1.01 & 1.02 & -0.13 \\
Ítem 50 & 2.66 & 1.15 & -0.06 & -1.47 \\
Ítem 51 & 2.08 & 1.15 & 0.65 & -1.05 \\
Ítem 52 & 3.10 & 1.05 & -0.70 & -0.91 \\
Ítem 53 & 1.92 & 1.03 & 0.92 & -0.32 \\
Ítem 54 & 1.81 & 0.98 & 1.09 & 0.11 \\
Ítem 55 & 3.24 & 0.99 & -0.94 & -0.45 \\
Ítem 56 & 2.93 & 1.10 & -0.43 & -1.27 \\
Ítem 57 & 1.37 & 0.77 & 2.26 & 4.41 \\
Ítem 58 & 2.13 & 1.02 & 0.63 & -0.67 \\
Ítem 59 & 3.42 & 0.91 & -1.33 & 0.46 \\
Ítem 60 & 1.94 & 1.04 & 0.84 & -0.50 \\
Ítem 61 & 1.57 & 0.86 & 1.55 & 1.61 \\
Ítem 62 & 2.06 & 1.11 & 0.68 & -0.91 \\
\hline
\end{tabular}

\section{Análisis de la estructura interna}

Para el examen de la estructura factorial se empleó el método de factorización de ejes principales, el cual es recomendado cuando no se cumplen los supuestos de normalidad multivariada (Fabrigar, Wegener, MacCallum \& Strahan, 1999) y se ha empleado en otros estudios sobre la escala (Méndez et al., 2002). El índice de adecuación muestral fue adecuado, Kaiser-Meyer-Olkin $(\mathrm{KMO})=.847$, y el test de esfericidad de Bartlett fue significativo $\left(\chi^{2}(1891,567)=7537.805, p=.000\right)$, lo cual señala la factibilidad del estudio factorial. Para la extracción de factores primero se utilizó la regla de Kaiser-Gutman, obteniéndose 19 factores con autovalores mayores a 1 . Debido a que dicha regla tiende a extraer demasiados factores, se utilizaron otros criterios. El gráfico de sedimentación sugirió la extracción de 3 factores, en tanto que el análisis paralelo de Horn 6 factores, por lo cual se analizaron soluciones 
factoriales de 3 a 6 factores. La estructura de 5 factores resultó la más simple y teóricamente coherente.

Siguiendo los lineamientos de Tabachnick y Fidell (2001), en un primer momento se realizó una rotación oblicua y se obtuvo la matriz de correlación entre los factores. Dado que se observaron correlaciones mayores a .32 entre los factores se continúo con la rotación oblicua (Promax). Cabe señalar que si bien la rotación ortogonal es más parsimoniosa y frecuentemente utilizada, en ciencias sociales generalmente existe algún grado de correlación entre los factores, por lo cual la rotación oblicua ofrece soluciones más precisas y reproducibles (Costelo \& Osborne, 2005). Sin embargo, dado que en otros estudios sobre el instrumento se empleó rotación Varimax (solución que supone factores no relacionados), se evaluó también la rotación ortogonal, obteniéndose una interpretación semejante de la solución factorial.

La matriz de configuración permite apreciar el aporte de cada factor a las puntuaciones individuales en cada una de las variables, es la matriz que habitualmente se considera cuando se utilizan rotaciones oblicuas. Como se observa en la tabla 2, 11 ítems presentan cargas factoriales menores a .30, sin embargo, se optó por conservarlos a los fines de facilitar comparaciones con otros estudios. En tanto que, al observar la matriz de estructura, la cual muestra las correlaciones entre los factores y las variables, varios ítems mostraron correlaciones con dos factores, guardando coherencia teórica (comportamientos sociales apropiados y no apropiados) (no se muestra aquí). Los cinco factores explicaron el $22.8 \%$ de la varianza total de comportamiento social. El Factor 1 comprende ítems referidos a Agresividad/Conducta Antisocial, el Factor 2 ítems referidos a Habilidades Sociales Apropiadas, el Factor 3 ítems sobre Amistad, el Factor 4 ítems referidos a Sobreconfianza/Celos/ Soberbia, y el Factor 5 contiene ítems sobre Soledad/Ansiedad Social 5 .

5 Un procedimiento distinto (exclusión de ítems con bajas cargas factoriales y comunalidad) dio lugar al agrupamiento de 44 ítems $(\alpha=.793)$ en tres factores que contenían ítems referidos a: Agresividad/Conducta Antisocial y Sobreconfianza/Celos/Soberbia $(\alpha=.86)$; Habilidades Sociales Apropiadas (con un ítem de Amistad) $(\alpha=.767)$; y Amistad $(\alpha=.628)$. Se puede acceder a estos resultados a través de la tercera autora. 


\section{Tabla 2}

Estructura factorial (matriz de configuración) de la escala MESSY-Autoinforme

\begin{tabular}{|c|c|c|c|c|c|c|}
\hline & \multicolumn{2}{|c|}{ Factores } & & \multicolumn{3}{|c|}{ Factores } \\
\hline & 1 & 2 & & 3 & 4 & 5 \\
\hline Ítem 22 & .628 & & Ítem 13 & .540 & & \\
\hline Ítem 5 & .616 & & Ítem 10 & .502 & & \\
\hline Ítem 30 & .503 & & Ítem 12 & .497 & & \\
\hline Ítem 3 & .502 & & Ítem 28 & .338 & & \\
\hline Ítem 11 & .474 & & Ítem 16 & .335 & & \\
\hline Ítem 21 & .454 & & Ítem 9 & .326 & & \\
\hline Ítem 14 & .451 & & Ítem 27 & .315 & & \\
\hline Ítem 2 & .426 & & Ítem 20 & .304 & & \\
\hline Ítem 35 & .416 & & Ítem 1 & .281 & & \\
\hline Ítem 4 & .413 & & Ítem 51 & & .613 & \\
\hline Ítem 7 & .401 & & Ítem 45 & & .444 & \\
\hline Ítem 19 & .400 & & Ítem 36 & & .403 & \\
\hline Ítem 8 & .398 & & Ítem 54 & & .399 & \\
\hline Ítem 62 & .395 & & Ítem 33 & & .369 & \\
\hline Ítem 61 & .390 & & Ítem 57 & & .342 & \\
\hline Ítem 17 & .379 & & Ítem 38 & & .212 & \\
\hline Ítem 15 & .368 & & Ítem 49 & & & .515 \\
\hline Ítem 29 & .357 & & Ítem 48 & & & .425 \\
\hline Ítem 18 & .336 & & Ítem 26 & & & .286 \\
\hline Ítem 6 & .323 & & Ítem 25 & & & .188 \\
\hline Ítem 53 & .312 & & & & & \\
\hline Ítem 60 & .292 & & & & & \\
\hline Ítem 39 & .268 & & & & & \\
\hline Ítem 41 & .262 & & & & & \\
\hline Ítem 37 & & .494 & & & & \\
\hline Ítem 56 & & .486 & & & & \\
\hline Ítem 46 & & .484 & & & & \\
\hline Ítem 44 & & .474 & & & & \\
\hline
\end{tabular}




\begin{tabular}{|c|c|c|c|c|c|}
\hline & \multicolumn{2}{|c|}{ Factores } & \multicolumn{3}{|c|}{ Factores } \\
\hline & 1 & 2 & 3 & 4 & 5 \\
\hline Ítem 40 & & .465 & & & \\
\hline Ítem 52 & & .462 & & & \\
\hline Ítem 59 & & .450 & & & \\
\hline Ítem 55 & & .449 & & & \\
\hline Ítem 47 & & .439 & & & \\
\hline Ítem 58 & & .363 & & & \\
\hline Ítem 34 & & .349 & & & \\
\hline Ítem 43 & & .337 & & & \\
\hline Ítem 50 & & .325 & & & \\
\hline Ítem 32 & & .315 & & & \\
\hline Ítem 31 & & .283 & & & \\
\hline Ítem 42 & & .283 & & & \\
\hline Ítem 23 & & .281 & & & \\
\hline Ítem 24 & & .257 & & & \\
\hline alfa de Cronbach & .85 & .79 & .69 & .65 & .43 \\
\hline $\begin{array}{l}\text { alfa de Cronbach } \\
\text { Total }\end{array}$ & .81 & & & & \\
\hline
\end{tabular}

\section{Consistencia interna}

La consistencia interna de cada factor y de la escala total se valoró a través del coeficiente alfa de Cronbach $(\alpha)$ : valores entre .70 y .80 se consideran buenas estimaciones de fiabilidad (Kaplan \& Sacuzzo, 2006), mientras que es aceptable un criterio menor (alrededor de .60) en escalas con escaso número de ítems y que cumplen los criterios señalados por Loewenthal (2001) (buena evidencia de validez, razones teóricas o prácticas o la escala es breve). La escala total mostró buena consistencia interna $(\alpha=.81)$, también los factores 1 (24 ítems, $\alpha=.85$ ) y 2 (18 ítems, $\alpha=.79$ ). En cambio los factores 3 (9 ítems, $\alpha=.69$ ) y 4 (7 ítems, $\alpha=.65$ ) evidenciaron índices aceptables, y el factor 5 un valor demasiado bajo (4 ítems, $\alpha=.43$ ). 


\section{Diferencias de sexo}

Finalmente, se evaluó la validez de la escala a través de la comparación de las puntuaciones de varones y mujeres en la puntuación total y cada una de las dimensiones de la escala, lo cual se realizó a través de pruebas $t$ de diferencias de medias, considerándose el promedio de los ítems, como se observa en la tabla 3. Los resultados del autoinforme del comportamiento social muestran que los varones presentan puntuaciones más altas en dos de las dimensiones negativas del comportamiento social: Agresividad/Conducta Antisocial $(t=4.27, p<.000)$ y Sobreconfianza/Celos/Soberbia $(t=2.13, p<.05)$, y también en la puntuación total de la escala $(t=4.63, p<.000)$, la cual señala comportamientos sociales no adaptativos. Por otra parte, las mujeres manifiestan un mejor desempeño social, con puntuaciones más altas en la dimensión de Habilidades Sociales Apropiadas $(t=-3.74, p<.000)$. Sin embargo, al considerar el tamaño del efecto, siguiendo los valores heurísticos propuestos por Cohen (1988), se aprecian valores pequeños, en la mayoría de los casos, como se observa en la tabla 3.

\section{Tabla 3}

Diferencias de sexo en las dimensiones y la puntuación total del MESSYAutoinforme

\begin{tabular}{|c|c|c|c|c|c|c|}
\hline \multirow[b]{2}{*}{ Factor } & \multicolumn{2}{|c|}{$\begin{array}{l}\text { Varones } \\
n=296\end{array}$} & \multicolumn{2}{|c|}{$\begin{array}{l}\text { Mujeres } \\
n=271 \\
\end{array}$} & \multirow[b]{2}{*}{$t$} & \multirow[b]{2}{*}{$\begin{array}{l}d \mathrm{de} \\
\text { Cohen }\end{array}$} \\
\hline & $M$ & $D E$ & $M$ & $D E$ & & \\
\hline Agresividad/Conducta Antisocial & 1.74 & 0.41 & 1.59 & 0.41 & $4.27^{* * *}$ & 0.37 \\
\hline Habilidades Sociales Apropiadas & 2.86 & 0.49 & 3.01 & 0.49 & $-3.74^{* * *}$ & -0.31 \\
\hline Amistad & 3.08 & 0.57 & 3.13 & 0.51 & -1.02 & -0.09 \\
\hline Sobreconfianza/Celos/Soberbia & 1.76 & 0.57 & 1.70 & 0.51 & $2.13^{*}$ & 0.11 \\
\hline Soledad/Ansiedad Social & 1.78 & 0.59 & 1.80 & 0.59 & -0.38 & -0.03 \\
\hline Escala Total & 1.46 & 0.30 & 1.34 & 0.31 & $4.63^{* * *}$ & 0.39 \\
\hline
\end{tabular}

${ }^{*} p<.05 .{ }^{* *} p<.01 .{ }^{* * *} p<.001$. 


\section{Comentario}

Este estudio se propuso evaluar las propiedades psicométricas de la versión de autoinforme de la escala MESSY. En términos generales, los resultados de validez y confiabilidad indican que es un instrumento adecuado para valorar el desempeño social en niños argentinos de 7 a 13 años, aunque son recomendables estudios posteriores.

Con respecto a la estructura factorial, se encontraron cinco dimensiones: Agresividad/Conducta Antisocial, Habilidades Sociales Apropiadas, Amistad, Sobreconfianza/ Celos/Soberbia, y Soledad/ Ansiedad Social. Los cinco factores explicaron el $22.8 \%$ de la varianza total de comportamiento social. Así, la cantidad de dimensiones resulta semejante a las señaladas en la versión original (Matson et al., 1983) y en la versión utilizada en esta investigación (Trianes et al., 2002). Sin embargo, algunos ítems cargaron en factores distintos a los propuestos en la versión original y también distintos a la versión en español. Incluso, en este estudio un conjunto de ítems que hacía referencia a los amigos se agrupó de manera notoria, dando lugar al factor Amistad que no había surgido en estudios anteriores. A pesar de ello, la estructura resultó teóricamente coherente, por lo que se respetó la pertenencia de los ítems a las dimensiones aquí observadas. Esto se realizó considerando también los hallazgos disímiles de diversas investigaciones sobre la estructura de la escala (Martins Teodoro et al., 2005; Méndez et al., 2002; Trianes et al., 2002), estudios donde también se ha observado una proporción de varianza explicada no demasiado elevada: $29.7 \%$ en Martins Teodoro et al., 2005, y 33.28\% en Méndez et al., 2002). Además, algunos ítems presentaban cargas factoriales inadecuadas, pero se los conservó con el fin de facilitar comparaciones con estudios de otros países.

En cuanto a la consistencia interna, tanto la escala total como los factores 1 y 2 mostraron índices adecuados $(\alpha=.81, .85$ y .79 , respectivamente), en tanto que los factores 3 y 4 evidenciaron índices aceptables $(\alpha=.69$ y .65 , respectivamente), y el factor 5 un valor demasiado bajo $(\alpha=.43)$. Futuros estudios podrían examinar en detalle los 
ítems comprendidos principalmente en el último factor, dado que la incorporación de nuevos ejemplares podría permitir obtener una imagen más precisa de la dimensión subyacente.

Asimismo, el presente estudio procuró obtener evidencia de validez de la escala a través de la comparación de las puntuaciones de varones y mujeres. Esto, considerando que la literatura sobre comportamiento social generalmente señala que los varones presentan más comportamientos agresivos y las niñas más comportamientos adaptativos (Bandeira, Rocha, Freitas, Del Prette \& Del Prette, 2006; Crick \& Grotpeter, 1995; Persson, 2005). Si bien se observaron diferencias estadísticamente significativas en algunas dimensiones y en la puntuación total de la escala MESSY, el tamaño del efecto fue reducido, resultado semejante al obtenido a partir de la versión docente (Ipiña et al., 2010).

Finalmente, cabe señalar posibles líneas de acción futuras. Este trabajo se enmarca en un estudio mayor sobre desempeńo social infantil, en el que se recurrió a docentes y niños como informantes. Sin embargo, y más allá de las cuestiones de orden práctico que generalmente conducen a emplear un reducido número de informantes, estudios posteriores podrían considerar la inclusión de los padres como informantes, procurando obtener un instrumento adecuado a nuestra población, y de esa manera contar con una imagen del comportamiento del niño en otro contexto. Por otra parte, también se recomienda incluir otros instrumentos de evaluación del comportamiento social, lo cual aportará evidencias de validez concurrente. Como señalan Reyna y Brussino (2009), se sugiere también desarrollar estudios de validez predictiva, considerando las repercusiones del comportamiento social temprano en los ámbitos sociales, académicos y de salud.

Luego del estudio realizado, se considera que la versión de autoinforme de la escala MESSY posee aceptables propiedades psicométricas, por lo cual se considera un buen instrumento para valorar el desempeño social de niños cordobeses de 7 a 13 años. Sin embargo, se sugiere la continuación de estudios en esta línea que confirmen la estructura obtenida, así como también indagar diversos aspectos relacionados con 
la validez del instrumento. Las diferencias en las soluciones encontradas remarcan la importancia de estudiar las propiedades psicométricas de los instrumentos elaborados en países de lengua y cultura diferentes a la población de interés (Poortinga, 2000).

\section{Referencias}

Bandeira, M., Rocha, S. S., Freitas, L. C., Del Prette, Z. A. \& Del Prette, A. (2006). Habilidades sociais e variáveis sociodemográficas em estudantes do ensino fundamental. Psicologia em Estudo, 11(3), 541-549.

Bank, L., Duncan, T., Patterson, G. R. \& Reid, J. (1993). Parent and teacher ratings in the assessment and prediction of antisocial and delinquent behaviors. Journal of Personality, 61, 693-709.

Brussino, S. A. (2002). Análisis causal del comportamiento agresivo infantil: pautas de crianza, estilo atribucional, capacidad intelectual y habilidad social (Tesis de doctorado inédita). Universidad Nacional de Córdoba, Argentina.

Chou, K. L. (1997). The Matson Evaluation of Social Skills with Youngsters: Reliability and validity of a Chinese translation. Personality and Individual Differences, 22, 123-125.

Cohen, J. (1988). Statistical power analysis for the behavioral sciences (2da. ed.). Mahwah, NJ: Lawrence Erlbaum.

Crick, N. R. \& Grotpeter, J. K. (1995). Relational aggression, gender, and social-psychological adjustment. Child Development, 66(3), 710-722.

Fabrigar, L. R., Wegener, D. T., MacCallum, R. C. \& Strahan, E. J. (1999). Evaluating the use of exploratory factor analysis in psychological research. Psychological Methods, 4, 272-299.

Goldstein, T. R., Miklowitz, D. J. \& Mullen, K. L. (2006). Social skills knowledge and performance among adolescents with bipolar disorder. Bipolar Disorder, 8, 350-361. 
Gresham, F. M., Sugai, G. \& Horner, R. H. (2001). Interpreting outcomes of social skills training for students with high-incidence disabilities. Exceptional Children, 67, 331-344.

Hair, J. F., Anderson, R. E., Tatham, R. L. \& Black, W. C. (1999). Análisis multivariante (5a. ed.). Madrid: Prentice Hall.

Ipiña, M. J., Molina, L. \& Reyna, C. (2010). Propiedades psicométricas de la Escala MESSY (versión docente) en una muestra de niños argentinos. Manuscrito presentado para su publicación.

Lacunza, A., Castro Solano, A. \& Contini de González, N. (2009). Habilidades sociales preescolares: una escala para niños en contextos de pobreza. Revista de Psicología, 27, 3-25.

Martins, T., Käpler, K., Martins de Freitas, P. \& Geraldi Haase, V. (2005). The Matson Evaluation of Social Skills with Youngsters (MESSY) and its adaptation for Brazilian children and adolescents. Revista Interamericana de Psicología, 39(2), 239-246.

Matson, J. L. \& Wilkins, J. (2009). Psychometric testing methods for children's social skills. Research in Developmental Disabilities: A Multidisciplinary Journal, 30(2), 249-274.

Matson, J. L., Rotatori, A. F. \& Helsel, W. J. (1983). Development of a rating scale to measure social skills in children: The Matson Evaluation of Social Skills with Youngsters (MESSY). Behaviour Research and Therapy, 21, 335-340.

Matson, J. L., Stabinsky-Compton, L. S. \& Sevin, J. A. (1991). Comparison and item analysis of the MESSY for autistic and normal children. Research in Developmental Disabilities, 12, 361-369.

Méndez, F., Hidalgo, M. \& Inglés, C. (2002). The Matson Evaluation of Social Skills with Youngsters. Psychometric properties of the Spanish translation in the adolescent population. European Journal of Psychological Assessment, 18(1), 30-42.

Parker, J. G. \& Asher, S. R. (1987). Peer relations and later personal adjustment: Are low-accepted children at risk? Psychological Bulletin, 102(3), 357-389.

Pereira Del Prette, Z. A. \& Del Prette, A. (2002). Psicología de las habilidades sociales: terapia y educación. México: Manual Moderno. 
Persson, G. E. (2005). Developmental perspectives on prosocial and aggressive motives in preschoolers' peer interactions. International Journal of Behavioral Development, 29(1), 80-91.

Poortinga, Y. H. (2000). Cross-cultural test adaptation. En A. E. Kazdin (Ed.), Encyclopedia of psychology (pp. 369-371). Washington, DC: American Psychological Association.

Reyna, C. E. \& Brussino, S. A. (2009). Propiedades psicométricas de las Escalas de Comportamiento Preescolar y Jardín Infantil en una muestra de niños argentinos de 3 a 7 años. Psykhe, 18(2), 127-140.

Rose-Krasnor, L. (1997). The nature of social competence: A theoretical review. Social Development, 6(1), 111-135.

Rubin, K. H., Dwyer, K. M., Booth-LaForce, C., Kim, A. H., Burgess, K. B. \& Rose-Krasnor, L. (2004). Attachment, friendship, and psychological functioning in early adolescence. Journal of Early Adolescence, 24, 326-356.

Schaeffer, C. M., Petras, H., Ialongo, N., Poduska, J. \& Kellam, S. (2003). Modeling growth in boys' aggressive behavior across elementary school: Links to later criminal involvement, conduct disorder, and anti-social personality disorder. Developmental Psychology, 39, 1020-1035.

Schulz, A. (2008). Validación de un sistema de evaluación de las habilidades sociales en niños argentinos por medio de informantes clave. Resultados preliminares (Informe final del Proyecto de Investigación 26/07 de la Facultad de Humanidades, Educación y Ciencias Sociales). Libertador San Martín, Argentina: Universidad Adventista del Plata.

Sharma, S., Sigafoos, J. \& Carroll, A. (2000). Social skills assessment of Indian children with visual impairments. Journal of Visual Impairments and Blindness, 94, 172-176.

Spence, S. H. \& Liddle, B. (1990). Self-report measures of social competence for children: An evaluation of social skills for youngsters and the List of Social Situation Problems. Behavioral Assessment, 12, 317-336. 
Tabachnick, B. \& Fidell, L. (2001). Using multivariate statistics (4ta. ed.). Nueva York: Harper \& Row.

Trianes, M. V., Blanca, M. J., Muñoz, A., García, B. \& Sánchez, A. (2003). Competencia social en alumnos con necesidades educativas especiales: nivel de inteligencia, edad y género. Revista de Psicologia General y Aplicada, 56(3), 325-338.

Trianes, M. V., Blanca, M. J., Muñoz, A., García, B., Cardelle-Elawar, M. \& Infante, L. (2002). Relaciones entre evaluadores de la competencia social en preadolescentes: profesores, iguales y autoinformes. Anales de Psicología, 18(2), 197-214.

Trianes, M. V., Muñoz, A. M. \& Jiménez, M. (1997). Competencia social: su educación y tratamiento. Madrid: Pirámide.

Recibido: 14 de diciembre, 2010 Aceptado: 27 de junio, 2011 